\title{
Discontinuous superlinear elliptic equations of divergence form
}

\author{
Dian K. Palagachev
}

Abstract. We prove existence and global Hölder regularity of the weak solution to the Dirichlet problem

$$
\begin{cases}\operatorname{div}\left(a^{i j}(x, u) D_{j} u\right)=b(x, u, D u) & \text { in } \Omega \subset \mathbb{R}^{n}, n \geq 2, \\ u=0 & \text { on } \partial \Omega \in C^{1} .\end{cases}
$$

The coefficients $a^{i j}(x, u)$ are supposed to be $V M O$ functions with respect to $x$ while the term $b(x, u, D u)$ allows controlled growth with respect to the gradient $D u$ and satisfies a sort of sign-condition with respect to $u$. Our results correct and generalize the announcements in Ragusa (Nonlinear Differ Equ Appl 13:605-617, 2007, Erratum in Nonlinear Differ Equ Appl 15:277-277, 2008).

Mathematics Subject Classification (2000). Primary 35J65, Secondary 35R05, 35B45, 35B65.

Keywords. Divergence form quasilinear elliptic equations, Weak solvability, Regularity, A priori estimates.

\section{Introduction}

The paper deals with solvability and regularity questions regarding the Dirichlet problem

$$
\begin{cases}D_{i}\left(a^{i j}(x, u) D_{j} u\right)=b(x, u, D u) & \text { in } \Omega, \\ u=0 & \text { on } \partial \Omega\end{cases}
$$

over a bounded domain $\Omega \subset \mathbb{R}^{n}, n \geq 2$, with $C^{1}$-smooth boundary. The data $a^{i j}(x, z)$ and $b(x, z, p)$ are assumed to be Carathéodory functions with $b(x, z, p)$ having controlled gradient growth, that is, $b(x, z, p)=\mathcal{O}\left(|p|^{r}\right)$ as $|p| \rightarrow \infty$ with

D. K. Palagachev was partially supported by the MIUR-PRIN project "Metodi variazionali ed equazioni differenziali non lineari". 
$r<(n+2) / n$ on the region where $b(x, z, p)$ and $z$ have the same sign, while $b(x, z, p)=\mathcal{O}(|p|)$ otherwise. For what concerns the coefficients $a^{i j}(x, z)$ of the uniformly elliptic operator, we allow these to be discontinuous in $x$ with discontinuity measured in terms of their appartenance to the space $V M O$ of functions with vanishing mean oscillations. We prove weak solvability of (1.1) in the framework of the Sobolev space $W_{0}^{1, q^{*}}(\Omega)$ where $q^{*}$ is the Sobolev conjugate of an exponent $q$ naturally appearing in the structure conditions satisfied by $b(x, z, p)$. It turns out that $q^{*}$ is always greater than the space dimension $n$ and this implies global Hölder continuity of the weak solution to (1.1) through the Morrey lemma. Indeed, the Hölder continuity of the essentially bounded solutions to the controlled growth problem (1.1) is not surprising being the essence of the celebrated De Giorgi-Nash breakthrough. What is the feature of our result is the explicit expression of the Hölder exponent in terms of $q$.

A problem like (1.1) with linear gradient growth in $b(x, z, p), b(x, z, p)=$ $\mathcal{O}(|p|)$, has been recently studied in [11] where the author, adapting our approach from [8], is looking for a weak solution to (1.1) as a fixed point of a suitable nonlinear operator $\mathcal{F}$ which should act from $W^{1, q}(\Omega)$ into itself. A delicate problem occurs with the missing compactness of $\mathcal{F}$, necessary in order to apply the Leray-Schauder fixed point principle, and this restricts the results in [11] to be valid only if $b(x, z, p)$ is independent of the gradient term $p, b(x, z, p)=b(x, z)$ (see the errata in [11]).

In order to prove weak solvability of (1.1) we derive a priori estimates in $L^{\infty}(\Omega)$ and in $W^{1,2}(\Omega)$ for any $W_{0}^{1,2}(\Omega)$-weak solution to (1.1). These estimates ensure the possibility to apply the Galerkin procedure in order to get existence of a weak solution $u \in W_{0}^{1,2}(\Omega)$. Starting from that solution and employing the structure assumptions on the data, we obtain the higher regularity $u \in W_{0}^{1, q^{*}}(\Omega)$ by means of interpolation of bootstrapping arguments. This approach relies on the well-posedness results regarding linear divergence form elliptic equations with $V M O$ coefficients and proved in $[3,4,10]$.

Actually, there exists a vast literature devoted to $W_{0}^{1,2}(\Omega)$-weak solvability of Dirichlet problems for divergence form uniformly elliptic operators with $L^{\infty}$ principal coefficients and with quadratic gradient growth at the first-order term, $b(x, z, p)=\mathcal{O}\left(|p|^{2}\right)$, under suitable structure condition on the data-let us only mention the deep papers $[1,2]$. The essence of our result here is that the $V M O$ regularity of the principal coefficients $a^{i j}(x, z)$ in $x$ and the controlled gradient growth $b(x, z, p)=\mathcal{O}\left(|p|^{r}\right), r<(n+2) / n$, imply higher integrability of the gradient $D u \in L^{q^{*}}(\Omega)$, that is, the weak solution of the problem (1.1) supports the Calderón-Zygmund property.

Finally, it is worth noting that the controlled growth condition $r<(n+2) / n$ is "almost optimal" for the validity of the results presented. In fact, growths strictly greater than $(n+2) / n$ would allow unbounded $W^{1,2}(\Omega)$-weak solutions as in the case of the equation $\Delta u=c|D u|^{R}$ which possesses the unbounded solution $u(x)=|x|^{\frac{2-R}{1-R}} \in W^{1,2}$ with $R \in\left(\frac{n+2}{n}, 2\right)$. The case of growth $r=(n+2) / n$ is 
more delicate and our bootstrapping approach fails now. Anyway, it was recently proved in [12] that any weak $W_{\text {loc }}^{1,2}(\Omega)$ solution to $(1.1)$ with $r=(n+2) / n$ is locally Hölder continuous with exactly the same Hölder exponent as the one here obtained. This makes us believe that our results will hold true in the limit case $r=(n+2) / n$ as well.

\section{Assumptions and main result}

Throughout the paper we will suppose $\Omega \subset \mathbb{R}^{n}, n \geq 2$, is a bounded domain with $C^{1}$-smooth boundary. The symbol $W^{1, q}(\Omega)$ stands, as usual, for the Sobolev space of once weakly differentiable functions $u: \Omega \rightarrow \mathbb{R}$, belonging to $L^{q}(\Omega)$ together with the gradient $D u$, and the standard summation convention on repeated indices is adopted. Let $a^{i j}(x, z): \Omega \times \mathbb{R} \rightarrow \mathbb{R}$ and $b(x, z, p): \Omega \times \mathbb{R} \times \mathbb{R}^{n} \rightarrow \mathbb{R}$ be Carathéodory functions which satisfy the following conditions:

- Strict ellipticity: there exists a positive constant $\lambda$ such that

$$
a^{i j}(x, z) \xi_{i} \xi_{j} \geq \lambda|\xi|^{2} \quad \text { a.a. } x \in \Omega, \quad \forall z \in \mathbb{R}, \quad \forall \xi \in \mathbb{R}^{n} .
$$

- Local uniform continuity of $a^{i j}$ in $z$, uniformly in $x$ : For each $M>0$ there exists a non-decreasing function $\mu_{M}:(0, \infty) \rightarrow(0, \infty)$ with $\lim _{t \downarrow} \mu_{M}(t)=0$ such that

$$
\left|a^{i j}(x, z)-a^{i j}\left(x, z^{\prime}\right)\right| \leq \mu_{M}\left(\left|z-z^{\prime}\right|\right) \quad \text { a.a. } x \in \Omega, \forall z, z^{\prime} \in[-M, M],
$$

and $a^{i j}(x, 0) \in L^{\infty}(\Omega)$.

- VMO regularity of $a^{i j}(x, z)$ with respect to $x$, locally uniformly in $z$ : For each $M>0$

$$
\sup _{|z| \leq M} \sup _{0<\rho \leq r} \sup _{x \in \Omega} \frac{1}{\left|\Omega_{\rho, x}\right|} \int_{\Omega_{\rho}, x}\left|a^{i j}(y, z)-\frac{1}{\left|\Omega_{\rho, x}\right|} \int_{\Omega_{\rho, x}} a^{i j}\left(y^{\prime}, z\right) d y^{\prime}\right| d y
$$

tends to zero as $r \downarrow 0$, where $\Omega_{\rho, x}=\{y \in \Omega:|y-x|<\rho\}$ and $\left|\Omega_{\rho, x}\right|$ is the Lebesgue measure of $\Omega_{\rho, x}$.

- Controlled gradient growth: There exist a non-decreasing function $\nu(t)>0$ and $b_{0} \in L^{q}(\Omega)$ with $q>n / 2$, such that

$$
|b(x, z, p)| \leq \nu(|z|)\left(b_{0}(x)+|p|^{r}\right) \quad \text { a.a. } x \in \Omega, \quad \forall(z, p) \in \mathbb{R} \times \mathbb{R}^{n},
$$

and $r<(n+2) / n$.

- Sign-condition of $b$ with respect to $z$ : There exist non-negative functions $b_{1}$ and $b_{2}$ with $b_{1}^{2}, b_{2} \in L^{q}(\Omega), q>n / 2$, such that

$$
-\operatorname{sign} z . b(x, z, p) \leq b_{1}(x)|p|+b_{2}(x) \quad \text { a.a. } x \in \Omega, \quad \forall(z, p) \in \mathbb{R} \times \mathbb{R}^{n} .
$$

Remark 2.1. 1. Let us note that (2.2) implies $a^{i j}(x, z) \in L^{\infty}(\Omega \times[-M, M])$ for each constant $M>0$ and

$$
\left\|a^{i j}\right\|_{L^{\infty}(\Omega \times[-M, M])} \leq C\left(M, \mu_{M},\left\|a^{i j}(\cdot, 0)\right\|_{L^{\infty}(\Omega)}\right) .
$$


In particular, the divergence form operator $D_{i}\left(a^{i j}(x, u) D_{j} u\right)$ is uniformly elliptic over the space of the essentially bounded functions.

2. For the sake of clarity, we propose the following example of principal coefficients satisfying the hypotheses $(2.1)-(2.3)$ :

$$
a^{i j}(x, z)=a_{1}^{i j}(x) f_{1}(z)+a_{2}^{i j}(x) \sin \left(|\log | x||^{\theta}\right) f_{2}(z)
$$

where $a_{k}^{i j}(x) \in C^{0}(\bar{\Omega}), k=1,2, a_{k}^{i j}(x) \xi_{i} \xi_{j} \geq \lambda_{k}|\xi|^{2}, \lambda_{1}>\lambda_{2}$, and $f_{k}(z) \in$ $C^{0}(\mathbb{R})$ are positive functions, bounded away from zero, $f_{k}(z) \geq m>0$. Regarding the exponent $\theta$, it belongs to the range $(0,1)$ in order to have $|\log | x||^{\theta} \in V M O$ (see [7, Chapter 2]) and it is supposed, of course, that $\Omega$ contains the origin.

For what concerns the first-order term $b(x, z, p)$, it is worth noting that (2.5) generalizes the classical sign-condition (cf. $[1,2]) z b(x, z, p) \geq 0$ which guarantees essential boundedness of the weak solutions to divergence form equations. The assumptions (2.4) and (2.5) are certainly satisfied when $b(x, z, p)$ has the sign of $z$ as happens, for instance, if

$$
b(x, z, p)=z^{2 k+1}|p|^{r}+b_{0}(x), \quad k \in \mathbb{N},
$$

or if

$$
b(x, z, p)=g\left(u^{+}\right)|p|^{r}-h\left(u^{-}\right)|p|+b_{0}(x),
$$

where $u^{ \pm}$stands for the positive/negative part of $u, g$ and $h$ are non-decreasing continuous functions such that $g(0)=h(0)=0$ and $h$ is bounded. Roughly speaking, (2.4) and (2.5) allow $b(x, u, D u)$ to grow as $|D u|^{r}$ in the set where $b$ and $u$ has the same sign, while linear gradient growth is assumed for $b$ otherwise.

3. The growth $r$ in the assumption (2.4) could be any non-negative number less than $(n+2) / n$. In fact, applying the Young inequality we may suppose, without loss of generality, that $r \geq 1$, or even $r \geq r_{0}$ for some $r_{0}<(n+2) / n$. In the sequel we will use this fact without explicit reference.

Let us recall that a function $u \in W_{0}^{1, s}(\Omega) \cap L^{\infty}(\Omega), s \geq 2$, is said to be a weak solution to the Dirichlet problem (1.1) if

$$
\int_{\Omega} a^{i j}(x, u(x)) D_{j} u(x) D_{i} \varphi(x) d x+\int_{\Omega} b(x, u(x), D u(x)) \varphi(x) d x=0
$$

for each $\varphi \in W_{0}^{1, s^{\prime}}(\Omega)$ where $s^{\prime}=s /(s-1)$. Indeed, having in mind $(2.4)$ and in order to ensure convergence of the second integral above, the exponent $s$ must be such that

$$
s \in \begin{cases}{\left[2, \frac{n q}{n-q}\right]} & \text { if } q<n, \\ {[2,+\infty)} & \text { if } q \geq n,\end{cases}
$$

while the assumption $u \in L^{\infty}(\Omega)$ gives $a^{i j}(x, u(x)) \in L^{\infty}(\Omega)$. 
The main result of the paper is the following

Theorem 2.2. Under the assumptions (2.1)-(2.5) the problem (1.1) admits a weak solution

$$
u \in W_{0}^{1, q^{*}} \cap L^{\infty}(\Omega) \quad \text { with } \quad q^{*}= \begin{cases}\frac{n q}{n-q} & \text { if } q<n, \\ \text { any number } \geq 1 & \text { if } q \geq n,\end{cases}
$$

satisfying the estimate

$$
\|u\|_{W^{1, q^{*}}(\Omega)}+\|u\|_{L^{\infty}(\Omega)} \leq C
$$

with $C$ depending on $n, \lambda, q, r,\left\|b_{0}\right\|_{L^{q}(\Omega)},\left\|b_{1}^{2}\right\|_{L^{q}(\Omega)},\left\|b_{2}\right\|_{L^{q}(\Omega)}$, the regularity of $\partial \Omega$ and on the Lebesgue measure $|\Omega|$. Moreover, this solution is a Hölder continuous function. Precisely, $u \in C^{0,2-n / q}(\bar{\Omega})$ if $q<n$ and $u \in C^{0, \alpha}(\bar{\Omega})$ for each $\alpha \in(0,1)$ when $q \geq n$.

It will be seen from the proof of Theorem 2.2 that (1.1) is weakly solvable in $W_{0}^{1,2}(\Omega) \cap L^{\infty}(\Omega)$ under the hypotheses $(2.1),(2.4)$ and (2.5). Later on, better regularity (2.2) and (2.3) of the principal coefficients $a^{i j}(x, z)$ ensures higher integrability $D u \in L^{q^{*}}(\Omega)$ of the gradient of every weak $W_{0}^{1,2}(\Omega) \cap L^{\infty}(\Omega)$ solution to (1.1), and its Hölder continuity follows from the Morrey lemma and the fact that $q^{*}>n$.

It is worth noting that the requirement $u \in L^{\infty}(\Omega)$ could be dropped from the definition of weak solution to the problem (1.1) by substituting (2.1) and (2.4) with

$$
\lambda^{-1}|\xi|^{2} \geq a^{i j}(x, z) \xi_{i} \xi_{j} \geq \lambda|\xi|^{2} \quad \text { a.a. } x \in \Omega, \forall z \in \mathbb{R}, \forall \xi \in \mathbb{R}^{n}
$$

and

$$
|b(x, z, p)| \leq a(x)\left(b_{0}(x)+|z|^{\frac{n+2}{n-2}}+|p|^{r}\right) \quad \text { a.a. } x \in \Omega, \forall(z, p) \in \mathbb{R} \times \mathbb{R}^{n},
$$

respectively, with arbitrary positive power of $|z|$ when $n=2$. The statement of Theorem 2.2 remains anyway true with $(2.1)^{\prime}$ and (2.4)' instead of (2.1) and (2.4). The details are left to the reader.

Let us point out that the assumptions (2.1)-(2.5) do not imply, in general, uniqueness for the weak solution from Theorem 2.2. However, some additional conditions on the data of (1.1) assure the unique solvability. For instance, (1.1) admits a unique weak solution if the coefficients $a^{i j}(x, z)$ are independent of $z$ and the nonlinear term $b(x, z, p)$ is Lipschitz continuous function with respect to $p$ which is non-decreasing in $z$ (cf. [5, Section 10.4] and [8, Theorem 1.4] for details). 


\section{Proof of Theorem 2.2}

First of all, we will prove that assumptions (2.1), (2.4) and (2.5) ensure weak solvability of (1.1) in $W_{0}^{1,2}(\Omega)$. For this goal, we will obtain a priori estimates in $L^{\infty}(\Omega)$ and in $W^{1,2}(\Omega)$ for each weak solution to the Dirichlet problem (1.1).

Proposition 3.1 ( $L^{\infty}$-a priori estimate) Assume (2.1) and (2.5) and let $u \in$ $W_{0}^{1,2}(\Omega) \cap L^{\infty}(\Omega)$ be a weak solution of (1.1). Then there is a constant $M=$ $M\left(n, \lambda, q,|\Omega|,\left\|b_{1}^{2}\right\|_{L^{q}(\Omega)},\left\|b_{2}\right\|_{L^{q}(\Omega)}\right)$ such that

$$
\|u\|_{L^{\infty}(\Omega)}=\sup _{x \in \Omega}|u(x)| \leq M .
$$

Proof. The estimate (3.1) is a direct consequence of the maximum principle for divergence form operators ([5, Theorem 10.9]) and the concluding remarks in [5, Chap. 10] regarding the integrability requirements for the functions $b_{1}$ and $b_{2}$. The reader is referred alternatively to [6, Chap. IV, Sect. 7].

Let us point out that the sign-condition (2.5) could be somewhat weakened by allowing dependence on $|z|$,

$$
-\operatorname{sign} z . b(x, z, p) \leq b_{1}(x)|p|+b_{2}^{\prime}(x)|z|+b_{2}(x) \quad \text { a.a. } x \in \Omega, \forall(z, p) \in \mathbb{R} \times \mathbb{R}^{n}
$$

with $b_{2}^{\prime} \in L^{q}(\Omega)$. For domains $\Omega$ of small enough Lebesgue measure this will give (cf. [5, Theorem 10.10]) the bound (3.1) with $M$ depending on $\left\|b_{2}^{\prime}\right\|_{L^{q}(\Omega)}$ in addition.

It is worth noting that (3.1) together with (2.1) and the gradient growth assumption (2.4) ensure coercivity of the form given by (2.6), that is,

$$
\int_{\Omega} a^{i j}(x, u) D_{j} u D_{i} u d x+\int_{\Omega} b(x, u, D u) u d x \geq C_{1}\|D u\|_{L^{2}(\Omega)}^{2}-C_{2} \quad \forall u \in W_{0}^{1,2}(\Omega)
$$

$C_{1}, C_{2}=$ const $>0$, which is equivalent to an a priori estimate in $W^{1,2}(\Omega)$ for each solution to (1.1). Here we prefer to derive directly that estimate.

Proposition 3.2 ( $L^{2}$-a priori estimate for the gradient) Assume (2.1), (2.4) and (2.5). There is a constant $K$ depending on the same quantities as $M$ in (3.1), and on $\nu(M)$ and $\left\|b_{0}\right\|_{L^{q}(\Omega)}$ in addition, such that

$$
\|D u\|_{L^{2}(\Omega)} \leq K
$$

for each $W_{0}^{1,2}(\Omega)$-weak solution of the problem (1.1).

Proof. In fact, $u \in L^{\infty}(\Omega)$ by means of Proposition 3.1. To derive (3.2) we set $\varphi=u$ as a test function in (2.6). The ellipticity condition (2.1) yields

$$
\lambda\|D u\|_{L^{2}(\Omega)}^{2} \leq \int_{\Omega} a^{i j}(x, u) D_{j} u D_{i} u d x,
$$


while

$$
\begin{aligned}
\int_{\Omega}|b(x, u, D u)||u| d x \leq & \int_{\Omega} \nu(|u|)|u|\left(b_{0}(x)+|D u|^{r}\right) d x \\
\leq & M \nu(M)\left(\left\|b_{0}\right\|_{L^{q}(\Omega)}|\Omega|^{\frac{q-1}{q}}+\|D u\|_{L^{2}(\Omega)}^{r}|\Omega|^{\frac{2-r}{2}}\right) \\
\leq & M \nu(M)\left(\left\|b_{0}\right\|_{L^{q}(\Omega)}|\Omega|^{\frac{q-1}{q}}+\varepsilon\|D u\|_{L^{2}(\Omega)}^{2}\right. \\
& \left.+\frac{(2-r) r^{\frac{r}{r-2}}}{2^{\frac{2 r-2}{r-2}}} \varepsilon^{-\frac{r}{2-r}}|\Omega|\right)
\end{aligned}
$$

after successive applications of (2.4) and (3.1), the Hölder and the Young inequalities and where $\varepsilon>0$ is arbitrary. This way,

$$
\lambda\|D u\|_{L^{2}(\Omega)}^{2} \leq \varepsilon M \nu(M)\|D u\|_{L^{2}(\Omega)}^{2}+C\left(\varepsilon, M, q, r,|\Omega|,\left\|b_{0}\right\|_{L^{q}(\Omega)}\right)
$$

and (3.2) follows by taking $\varepsilon=\frac{\lambda}{2 M \nu(M)}$.

With the a priori bounds (3.1) and (3.2) at hand, it is standard to get weak solvability in $W_{0}^{1,2}(\Omega)$ of the problem (1.1). In fact, it suffices to apply the Galerkin procedure as done in [6, Chap. IV, Sect. 9]. Precisely, Theorem 9.2 of $\left[6\right.$, Chap. IV] yields. ${ }^{1}$

Proposition $3.3\left(W_{0}^{1,2}(\Omega)\right.$-weak solvability) Under the hypotheses $(2.1),(2.4)$ and (2.5), the Dirichlet problem (1.1) is weakly solvable in $W_{0}^{1,2}(\Omega) \cap L^{\infty}(\Omega)$ with solution satisfying the bound

$$
\|u\|_{W^{1,2}(\Omega)}+\|u\|_{L^{\infty}(\Omega)} \leq C,
$$

where $C=C\left(n, \lambda, q, r,|\Omega|,\left\|b_{0}\right\|_{L^{q}(\Omega)},\left\|b_{1}^{2}\right\|_{L^{q}(\Omega)},\left\|b_{2}\right\|_{L^{q}(\Omega)},\left\|a^{i j}(\cdot, 0)\right\|_{L^{\infty}(\Omega)}\right)$.

Turning back to the proof of our main result, Theorem 2.2, and having in mind Proposition 3.3, it remains to get the higher regularity $u \in W^{1, q^{*}}(\Omega)$ for the weak $W_{0}^{1,2}(\Omega) \cap L^{\infty}(\Omega)$ solution of the problem (1.1).

To start with, let us note that the ellipticity condition (2.1) together with the controlled growth assumption (2.4), $r<2$, and (3.1) ensure Hölder continuity of $u$ in $\bar{\Omega}$ through [6, Chapter IV, Theorem 1.1]. Precisely,

$$
\sup _{x, y \in \bar{\Omega}} \frac{|u(x)-u(y)|}{|x-y|^{\gamma}} \leq C\left(M,\left\|b_{0}\right\|_{L^{q}(\Omega)}, \partial \Omega\right)
$$

with some exponent $\gamma=\gamma\left(M,\left\|b_{0}\right\|_{L^{q}(\Omega)}, \partial \Omega\right) \in(0,1)$. We invoke now our result from [8, Lemma 2.1] (see also [7, Lemma 2.6.2] or [9, Lemma 1]) to conclude that

$$
A^{i j}(x)=a^{i j}(x, u(x)) \in V M O(\Omega) \cap L^{\infty}(\Omega)
$$

\footnotetext{
${ }^{1}$ In order to ensure the requirements of [6, Chapter IV, Theorem 9.2] one must have $r \geq 4 / n$ when $n>2$. Indeed, this condition is not restrictive and could be imposed in (2.4) at the very beginning as Remark 2.1 notes.
} 
on the base of (2.2) and (2.3), with $V M O$-modulus of the composition $A^{i j}$ bounded in terms of $M, \mu_{M}(M)$ and $\left\|a^{i j}(\cdot, 0)\right\|_{L^{\infty}(\Omega)}$.

Fixed the solution $u \in W_{0}^{1,2}(\Omega)$ of (1.1), let

$$
f(x)=b(x, u(x), D u(x)) .
$$

This linearizes the problem (1.1) into

$$
\begin{cases}D_{i}\left(A^{i j}(x) D_{j} u\right)=f(x) & \text { in } \Omega, \\ u=0 & \text { on } \partial \Omega\end{cases}
$$

with uniformly elliptic coefficients matrix $\left\{A^{i j}(x)\right\}_{i, j=1}^{n}$ of $V M O(\Omega) \cap L^{\infty}(\Omega)$ entries. The desired higher integrability of the gradient $D u$ will be derived on the base of the regularity theory for linear divergence form elliptic equations with $V M O$ principal coefficients.

Lemma 3.4. Let $\Omega$ be a bounded and $C^{1}$-smooth domain in $\mathbb{R}^{n}, n \geq 2$, and suppose $\left\{A^{i j}(x)\right\}_{i, j=1}^{n}$ is a real and uniformly elliptic matrix of measurable entries belonging to $\operatorname{VMO}(\Omega) \cap L^{\infty}(\Omega)$.

Then the Dirichlet problem (3.4) is uniquely solvable in $W_{0}^{1, s^{*}}(\Omega)$ for all $f \in L^{s}(\Omega)$ and all $s>1$, where

$$
s^{*}= \begin{cases}\frac{n s}{n-s} & \text { if } s<n, \\ \text { any number } \geq 1 & \text { if } s \geq n .\end{cases}
$$

Moreover,

$$
\|D u\|_{L^{s^{*}}(\Omega)} \leq C\|f\|_{L^{s}(\Omega)}
$$

with a constant $C$ depending on $n, s$, the ellipticity constant and the $L^{\infty}$-norms of $A^{i j}$, their VMO-moduli, $\partial \Omega$ and $|\Omega|$.

Proof. Making use of the well-known imbedding of $L^{s}(\Omega)$ into $W^{-1, s^{*}}(\Omega)$, there exists a vector field $\mathbf{F} \in\left(L^{s^{*}}(\Omega)\right)^{n}$ such that

$$
f(x)=\operatorname{div}(\mathbf{F}(x)) \quad \text { a.a. } x \in \Omega
$$

and

$$
\|\mathbf{F}\|_{\left(L^{s^{*}}(\Omega)\right)^{n}} \leq C(n, s, \partial \Omega)\|f\|_{L^{s}(\Omega)} .
$$

This way, the problem (3.4) takes on the form

$$
\begin{cases}D_{i}\left(A^{i j}(x) D_{j} u\right)=\operatorname{div}(\mathbf{F}(x)) & \text { in } \Omega, \\ u=0 & \text { on } \partial \Omega\end{cases}
$$

and we are in a position to apply the regularity theory of linear equations.

It was proved by Di Fazio in [4] that $A^{i j} \in V M O \cap L^{\infty}, A^{i j}=A^{j i}$ and $\partial \Omega \in C^{1,1}$ are sufficient conditions ensuring unique weak solvability of (3.7) in $W_{0}^{1, p}(\Omega)$ for all $\mathbf{F} \in\left(L^{p}(\Omega)\right)^{n}$ and all $p \in(1, \infty)$. Moreover, (3.7) supports the $L^{p}$-regularizing property, that is, $u \in W_{0}^{1, p}(\Omega)$ and $\mathbf{F} \in\left(L^{q}(\Omega)\right)^{n}$ with $q>p$ yield 
$u \in W_{0}^{1, q}(\Omega)$. These results were extended by Qafsaoui and Auscher in [10] to the case of non-symmetric operators over $C^{1}$-smooth domains and, more recently, by Byun [3] to the case of domains with sufficiently flat Lipschitz boundaries (like $C^{1}$, for instance) and for $A^{i j}$ belonging to the space of functions with small $B M O$ norms which contains $V M O$ as a proper subspace.

Therefore, the claim of Lemma 3.4 follows from [10, Theorem 1] or [3, Theorem 1.5] and (3.6).

Turning back to the proof of Theorem 2.2, we will apply the regularizing result from Lemma 3.4 to (3.4) through interpolation of bootstrapping arguments in order to get the higher integrability of the gradient.

We have $|D u|^{r} \in L^{2 / r}(\Omega)$ since $u \in W^{1,2}(\Omega)$ and therefore (3.3), (3.1) and (2.4) imply $f \in L^{q_{1}}(\Omega)$ with

$$
q_{1}=\min \left\{q, \frac{2}{r}\right\} .
$$

It follows from Lemma 3.4 applied to (3.4) that

$$
D u \in L^{q_{1}^{*}}(\Omega) .
$$

At this point let us stress reader's attention on the fact that the claim of Theorem 2.2 is immediate always when either $q \leq 2 / r$ or $2 / r \geq n$ because in both cases one has $q_{1}^{*}=q^{*}$. In fact, $q \leq 2 / r$ means $q_{1}=q$, that is, $q_{1}^{*}=q^{*}$. If instead $2 / r \geq n$ then we have two possibilities: either $q_{1}=q$ and we are done, or $q_{1}=2 / r$ whence $q>2 / r \geq n$ and $q_{1}^{*}=(2 / r)^{*}=q^{*}$ once again. This way, the essential case to be considered is

$$
\frac{2}{r}<q \text { and } \frac{2}{r}<n, \text { that is, } q_{1}=\frac{2}{r}<n .
$$

We have $q_{1}^{*}=(2 / r)^{*}=\frac{2 n}{n r-2}$ now and therefore (3.8) reads

$$
D u \in L^{\frac{2 n}{n r-2}}(\Omega) .
$$

Proceeding as above, $|D u|^{r} \in L^{\frac{2 n}{n r^{2}-2 r}}(\Omega)$ and (3.3), (3.1) and (2.4) give $f \in$ $L^{q_{2}}(\Omega)$ with

$$
q_{2}=\min \left\{q, \frac{2 n}{n r^{2}-2 r}\right\}
$$

and

$$
D u \in L^{q_{2}^{*}}(\Omega)
$$

by virtue of Lemma 3.4 and (3.4).

The cases $q \leq \frac{2 n}{n r^{2}-2 r}$ or $\frac{2 n}{n r^{2}-2 r} \geq n$ complete immediately the proof of Theorem 2.2 since $q_{2}^{*}=q^{*}$ and therefore the essential situation to deal with is

$$
\frac{2 n}{n r^{2}-2 r}<q \text { and } \frac{2 n}{n r^{2}-2 r}<n, \quad \text { that is, } \quad q_{2}=\frac{2 n}{n r^{2}-2 r}<n \text {. }
$$


One has $q_{2}^{*}=\left(\frac{2 n}{n r^{2}-2 r}\right)^{*}=\frac{2 n}{n r^{2}-2 r-2}$ and this rewrites (3.9) as

$$
D u \in L^{\frac{2 n}{n r^{2}-2 r-2}}(\Omega) .
$$

The $k$ th iteration of this procedure gives $f \in L^{q_{k}}(\Omega)$,

$$
q_{k}=\min \left\{q, \frac{2 n}{n r^{k}-2 r^{k-1}-2 r^{k-2}-\cdots-2 r}\right\},
$$

whence

$$
D u \in L^{q_{k}^{*}}(\Omega)
$$

as result of Lemma 3.4 and (3.4).

To reach our goal to derive (2.7), we will show that there is a large enough integer $k=k(n, r)$ for which

$$
\frac{2 n}{n r^{k}-2 r^{k-1}-2 r^{k-2}-\cdots-2 r} \geq n \text {. }
$$

In fact, $q_{k}=q$ would give $q_{k}^{*}=q^{*}$ whence (2.7) on the base of (3.10). Otherwise, $q_{k}=\frac{2 n}{n r^{k}-2 r^{k-1}-\cdots-2 r}<q$ but with (3.11) at hand we get $q \geq n$ whence $q_{k}^{*}=q^{*}$ once again.

To prove (3.11), we note that it is equivalent to

$$
n r^{k}-2 r^{k-1}-2 r^{k-2}-\cdots-2 r-2 \leq 0
$$

for the fixed growth $r \in\left(1, \frac{n+2}{n}\right)$ from (2.4) and some $k \in \mathbb{N}$ to be chosen. Indeed, the right-hand side of (3.12) equals

$$
\frac{r^{k}[n r-(n+2)]+2}{r-1}=\frac{r^{k} N(r)+2}{r-1}, \quad N(r)=n r-(n+2),
$$

and we have $N(r)<0$ while $\lim _{k \rightarrow+\infty} r^{k}=+\infty$. Thus, taking $k$ to be the smallest integer $k \geq \frac{\log \frac{2}{n+2-n r}}{\log r}$ we get the quantity in (3.13) negative whence (3.12) and thus (3.11).

To complete the proof of Theorem 2.2, it remains to note that the estimate (2.8) follows from the a priori bounds (3.1), (3.2) and (3.5), while the Hölder continuity of the weak solution to (1.1) is an immediate consequence of the fact that $q^{*}>n$, the Sobolev imbedding theorem and the Morrey lemma.

Remark 3.5. For the sake of comparison with what is really proved in [11], let us note that the statement of Theorem 2.2 is trivial when $b(x, z, p)=b(x, z)$. In fact, the problem (1.1) rewrites then as

$$
\begin{cases}D_{i}\left(a^{i j}(x, u) D_{j} u\right)=b(x, u) & \text { in } \Omega, \\ u=0 & \text { on } \partial \Omega\end{cases}
$$

while (2.4) and (2.5) take on the forms

$$
|b(x, z)| \leq \nu(|z|) . b_{0}(x) \quad \text { a.a. } x \in \Omega, \forall z \in \mathbb{R}, b_{0} \in L^{q}(\Omega), q>n / 2,
$$


and

$$
-\operatorname{sign} z . b(x, z) \leq b_{2}(x) \quad \text { a.a. } x \in \Omega, \forall z \in \mathbb{R}, b_{2} \in L^{q}(\Omega), q>n / 2
$$

respectively. Once having the $L^{\infty}(\Omega)$-estimate $(3.1),(2.4)^{\prime \prime}$ gives $b(x, u(x)) \in$ $L^{q}(\Omega)$ with $q>n / 2$ and the celebrated Hölder continuity result of De Giorgi ensures $u \in C^{0, \gamma}(\Omega)$ for some $\gamma \in(0,1)$. This way, $a^{i j}(x, u(x)) \in V M O(\Omega) \cap L^{\infty}(\Omega)$ by virtue of [8, Lemma 2.1] and $u \in W^{1, q^{*}}(\Omega)$ follows from Lemma 3.4 applied to $(1.1)^{\prime \prime}$.

\section{Acknowledgments}

Special thanks are due to the referee for the careful reading of the manuscript and the valuable comments and suggestions.

\section{References}

[1] Boccardo, L., Murat, F., Puel, J.P.: Existence de solutions non bornées pour certaines équations quasi-linéaires. Port. Math. 41, 507-534 (1982)

[2] Boccardo, L., Murat, F., Puel, J.P.: $L^{\infty}$ estimate for some nonlinear elliptic partial differential equations and application to an existence result. SIAM J. Math. Anal. 23, 326-333 (1992)

[3] Byun, S.-S.: Elliptic equations with BMO coefficients in Lipschitz domains. Trans. Am. Math. Soc. 357, 1025-1046 (2005)

[4] Di Fazio, G.: $L^{p}$-estimates for divergence form elliptic equations with discontinuous coefficients. Boll. Unione Mat. Ital. VII. Ser. A 10, 409-420 (1996)

[5] Gilbarg, D., Trudinger, N.S.: Elliptic Partial Differential Equations of Second Order, 3rd edn. Springer, Berlin (1997)

[6] Ladyzhenskaya, O.A., Ural'tseva, N.N.: Linear and Quasilinear Equations of Elliptic Type (in Russian), 2nd edn. Nauka, Moscow (1973)

[7] Maugeri, A., Palagachev, D.K., Softova, L.G.: Elliptic and Parabolic Equations with Discontinuous Coefficients. Wiley-VCH, Berlin (2000)

[8] Palagachev, D.K.: Quasilinear elliptic equations with VMO coefficients. Trans. Am. Math. Soc. 347, 2481-2493 (1995)

[9] Palagachev, D.K., Recke, L., Softova, L.G.: Applications of the differential calculus to nonlinear elliptic operators with discontinuous coefficients. Math. Ann. 336, 617-637 (2006)

[10] Qafsaoui, M., Auscher, P.: Observations on $W^{1, p}$ estimates for divergence elliptic equations with VMO coefficients. Boll. Unione Mat. Ital. Sez. B Artic. Ric. Mat. 5(8), 487-509 (2002) 
[11] Ragusa, M.A.: Linear growth coefficients in quasilinear equations. Nonlinear Differ. Equ. Appl. 13 (2007), 605-617; Nonlinear Differ. Equ. Appl. 15, 277-277 (Erratum) (2008)

[12] Zheng, S., Feng, Z.: Regularity for quasi-linear elliptic systems with discontinuous coefficients. Dyn. Partial Differ. Equ. 5, 87-99 (2008)

D. K. Palagachev

Dipartimento di Matematica

Politecnico di Bari

Via E. Orabona, 4

70125 Bari, Italy

e-mail: dian@dm.uniba.it; palaga@poliba.it

Received: 8 July 2008.

Accepted: 18 June 2009. 